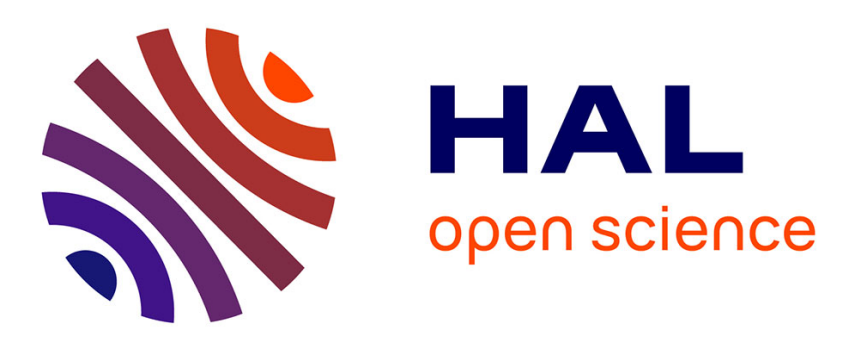

\title{
Relaxed conditions for the stability of switched nonlinear triangular systems under arbitrary switching
}

Ndolane Sene, Antoine Chaillet, Moussa Balde

\section{To cite this version:}

Ndolane Sene, Antoine Chaillet, Moussa Balde. Relaxed conditions for the stability of switched nonlinear triangular systems under arbitrary switching. Systems and Control Letters, 2015, 84, pp.5256. 10.1016/j.sysconle.2015.06.004 . hal-01264969

\section{HAL Id: hal-01264969 \\ https://hal-centralesupelec.archives-ouvertes.fr/hal-01264969}

Submitted on 3 Mar 2020

HAL is a multi-disciplinary open access archive for the deposit and dissemination of scientific research documents, whether they are published or not. The documents may come from teaching and research institutions in France or abroad, or from public or private research centers.
L'archive ouverte pluridisciplinaire HAL, est destinée au dépôt et à la diffusion de documents scientifiques de niveau recherche, publiés ou non, émanant des établissements d'enseignement et de recherche français ou étrangers, des laboratoires publics ou privés. 


\title{
Relaxed conditions for the stability of switched nonlinear triangular systems under arbitrary switching ${ }^{\text {th }}$
}

\author{
Ndolane Sene ${ }^{\mathrm{a}}$, Antoine Chaillet ${ }^{\mathrm{b}, *}$, Moussa Balde $^{\mathrm{a}}$ \\ ${ }^{a}$ Dept. Maths et Info, FST, UCAD, Dakar, Senegal. \\ ${ }^{b}$ Univ. Paris Sud-L2S - CentraleSupélec. 3, rue Joliot-Curie. 91192, Gif sur Yvette, \\ France.
}

\begin{abstract}
This paper addresses the stability of a class of nonlinear switching systems under arbitrary switching. It focuses on switching systems whose modes are made of a cascade of nonlinear scalar systems. It relaxes the stability conditions proposed by Angeli and Liberzon, by relying on the Strong iISS property which is known to be preserved under cascade interconnection. Its applicability is illustrated by the study of switched bilinear systems in triangular form.
\end{abstract}

Keywords: Nonlinear switching systems, Triangular systems, Stability analysis, Bilinear systems

\section{Introduction}

Providing conditions under which a switching system is globally asymptotically stable under arbitrary switching (UGAS) has been the object of intense research. It is well known that switching among nonlinear dynamics that share 5 a common Lyapunov function is UGAS. For linear time-invariant (LTI) systems, UGAS is guaranteed if the state matrices of the individual modes are Hurwitz and either i) commute with one another, ii) are symmetric, or iii) are normal:

\footnotetext{
This research was partially supported by a public grant overseen by the French ANR as part of the "Investissements d'Avenir" program, through the iCODE institute, research project funded by the IDEX Paris-Saclay, ANR-11-IDEX-0003-02.

* Corresponding author.

Email addresses: ndolanesene@yahoo.fr (Ndolane Sene), antoine.chaillet@centralesupelec.fr (Antoine Chaillet), moussa.balde.math@ucad.edu.sn (Moussa Balde)
}

Preprint submitted to Systems and Control Letters

June 4, 2015 
see [1, 2] and references therein for more details. See also [3] for a thorough study of the 2-dimensional case.

A powerful way to study stability of a high-order dynamical system is to see it as the interconnection of simpler dynamical systems. In general, this interconnection is bidirectional, in which case results such as the small-gain theorem have to be used in order to infer the stability of the overall system [4]. However, the complexity of the analysis is dramatically reduced when the 15 subsystems are interconnected in a unidirectional way; in other words, when the overall system is in triangular form [5, 6, 8, 7]. It is well known that the switching among LTI systems with Hurwitz triangular matrices is UGAS. This feature has been extended in [9] to nonlinear switched systems. It was shown, in particular, that if the individual modes are all made of a cascade of scalar 20 subsystems, then GAS is preserved under arbitrary switching provided that the subsystems are input-to-state stable (ISS, [10]) with respect to the state of the driving subsystems. The proof of this result relies on the property that ISS is preserved under cascade interconnection [11].

Recently, a less conservative property than ISS has been introduced, which still retains the feature of being preserved under cascade interconnection [12]. This property is known as the Strong iISS, and constitutes an interesting compromise between the generality of integral-ISS (iISS, [13]) and the strength of ISS. Strong iISS was introduced in [14, together with Lyapunov tools to ensure it in practice.

The goal of this paper is therefore to relax the conditions of [9] under which triangular switched systems are UGAS, by requiring that each stage of the triangular system is Strongly iISS rather than ISS. This relaxation extends the class of systems that can be addressed with this technique. It allows for instance to consider specific classes of switching systems whose individual modes are 35 stabilized by saturated feedback [15], and allows to cope with systems whose dynamics switches among bilinear systems.

The main challenge in relaxing the conditions of [9] by exploiting the Strong iISS property stands in the fact that no characterization of Strong iISS involving 
a single Lyapunov function has yet been established (see [14 for a counterexample on a natural conjecture to that regards). Consequently, we need to rely either on solution based reasoning, or on the existing Lyapunov characterizations for ISS [16] and iISS [17].

The paper is organized as follows: after recalling some necessary definitions, we describe the class of nonlinear switched systems considered here and provide our main result (Section 2), we then apply this result to switched bilinear systems (Section 3). All proofs are provided in Section 4

Notation. Given $\rho \in \mathbb{N}_{\geq 1}, \mathcal{P}$ denotes the set of all piecewise constant signals $\sigma: \mathbb{R}_{\geq 0} \rightarrow P:=\{1, \ldots, \rho\}$ that admit a finite number of discontinuities over any finite time interval. $\mathcal{P} \mathcal{D}$ denotes the set of all continuous functions $\alpha: \mathbb{R}_{\geq 0} \rightarrow \mathbb{R}_{\geq 0}$ satisfying $\alpha(0)=0$ and $\alpha(s)>0$ for all $s>0$. A class $\mathcal{K}$ function is an increasing $\mathcal{P D}$ function. The class $\mathcal{K}_{\infty}$ denotes the set of all unbounded $\mathcal{K}$ functions. A continuous function $\beta: \mathbb{R}_{\geq 0} \times \mathbb{R}_{\geq 0} \longrightarrow \mathbb{R}_{\geq 0}$ is said to be of class $\mathcal{K} \mathcal{L}$ if $\beta(\cdot, t) \in \mathcal{K}$ for any $t \in \mathbb{R}_{\geq 0}$ and $\beta(s, \cdot)$ is non-increasing and tends to zero as its argument tends to infinity. Given $x \in \mathbb{R}^{n},|x|$ stands for its Euclidean norm: $|x|:=\sqrt{x_{1}^{2}+\ldots+x_{n}^{2}}$. Given $\epsilon>0, \operatorname{sat}_{\epsilon}(s):=\operatorname{sign}(s) \min \{\epsilon,|s|\}$ for all $s \in \mathbb{R}$. Given $m \in \mathbb{N}_{\geq 1}$, $\operatorname{sat}_{\epsilon}^{m}(d):=\left(\operatorname{sat}_{\epsilon}\left(d_{1}\right), \ldots, \text { sat }_{\epsilon}\left(d_{m}\right)\right)^{T}$ for all $d \in \mathbb{R}^{m}$. $\mathcal{U}^{m}$ denotes the set of all measurable, locally essentially bounded functions $u$ : $\mathbb{R}_{\geq 0} \rightarrow \mathbb{R}^{m}$. Given $u \in \mathcal{U}^{m},\|u\|:=\operatorname{ess} \sup _{t \geq 0}|u(t)|$.

\section{Problem statement and main result}

\subsection{Preliminary definitions}

The present paper studies the stability of a particular class of switched nonlinear systems. Generally speaking, a switched nonlinear system is defined as

$$
\dot{x}=f_{\sigma}(x)
$$

where $\sigma \in \mathcal{P}$ and, for each $p \in P, f_{p}: \mathbb{R}^{n} \rightarrow \mathbb{R}^{n}$ denotes a locally Lipschitz function. This system is said to be uniformly globally asymptotically stable 
(UGAS) if there exists a $\mathcal{K} \mathcal{L}$ function $\beta$ such that, for all $x^{0} \in \mathbb{R}^{n}$ and all $\sigma \in \mathcal{P}$, its solution satisfies $\left|x\left(t ; x^{0}, \sigma\right)\right| \leq \beta\left(\left|x^{0}\right|, t\right)$ for all $t \geq 0$.

Similarly to [9], this paper exploits specific input-to-state properties of the individual subsystems to derive stability of the overall switched system. This requires us to consider switched systems with inputs:

$$
\dot{x}=f_{\sigma}(x, u)
$$

where $u \in \mathcal{U}^{m}$ represents the exogenous input. This system is said to be uniformly input-to-state stable with respect to small inputs (ISS wrt small inputs) if there exist a positive constant $R$, called input threshold, $\beta \in \mathcal{K} \mathcal{L}$, and $\gamma \in \mathcal{K}_{\infty}$ such that, for all $x^{0} \in \mathbb{R}^{n}$, all $\sigma \in \mathcal{P}$, and all $u \in \mathcal{U}^{m}$, its solution satisfies

$$
\|u\| \leq R \quad \Rightarrow \quad\left|x\left(t ; x^{0}, \sigma, u\right)\right| \leq \beta\left(\left|x^{0}\right|, t\right)+\gamma(\|u\|), \quad \forall t \geq 0 .
$$

If the right-hand side of the above implication holds for all $u \in \mathcal{U}^{m}$, we recover the notion of input-to-state stability (ISS) introduced in [18] and widely studied in the literature: see [10] for a survey. ISS wrt small inputs thus requires ISS as long as the input amplitude does not overpass the input threshold $R$. It should not be confused with the notion of local ISS (LISS), used for instance in [19], for which the above estimate is required to hold only for small initial conditions and small input amplitudes: on the contrary, ISS wrt small inputs constrains the input magnitude but not the initial state. Note that ISS wrt to small inputs implies UGAS of the switched system in the absence of exogenous inputs $(u=0)$. It also guarantees a bounded response to any input whose amplitude is below the input threshold $R$. Nonetheless, it provides no information on the behavior of the system when the applied input has an amplitude greater than $R$ : solutions may diverge or even escape in finite time. To prevent this, the Strong iISS property introduced in [14] combines it with integral input-to-state stability (iISS, [13). More precisely, we say that (1) is Strongly iISS if it is both ISS wrt to small inputs and iISS, the latter meaning that there exist $\beta \in \mathcal{K} \mathcal{L}$ and $\eta_{1}, \eta_{2} \in \mathcal{K}_{\infty}$ such that, for all $x^{0} \in \mathbb{R}^{n}$, all $u \in \mathcal{U}^{m}$, and all $\sigma \in \mathcal{P}$,

$$
\left|x\left(t ; x^{0}, \sigma, u\right)\right| \leq \beta\left(\left|x^{0}\right|, t\right)+\eta_{1}\left(\int_{0}^{t} \eta_{2}(|u(s)|) d s\right), \quad \forall t \geq 0 .
$$


${ }_{65}$ By combining iISS and ISS wrt small inputs, the Strong iISS property thus ensures existence of solutions at all times, bounded state in response to any input signal whose amplitude is below the input threshold $R$, and a vanishing state in response to any vanishing input: see [14] for further details.

We stress that the extension of these properties to non-switched systems can be straightforwardly deduced by the above definitions by considering that $\sigma$ can only take a single value (namely, $P=\{1\}$ ).

\subsection{Problem statement}

While Strong iISS a much weaker robustness property than ISS, they share a crucial common feature: they are both preserved under cascade interconnection 14, 11. This feature was extensively exploited in [9] to analyze the stability of switched nonlinear systems in triangular form. The objective of this paper is to generalize this result, by replacing the ISS requirement by the more general Strong iISS property.

Let us start by formally introducing the class of systems considered here. Given $n \in \mathbb{N}_{\geq 1}$ we address the following switched dynamics :

$$
\left(\begin{array}{c}
\dot{x}_{1} \\
\vdots \\
\dot{x}_{i} \\
\vdots \\
\dot{x}_{n-1} \\
\dot{x}_{n}
\end{array}\right)=\left(\begin{array}{c}
f_{\sigma}^{1}\left(x_{1}, x_{2 \rightarrow n}\right) \\
\vdots \\
f_{\sigma}^{i}\left(x_{i}, x_{i+1 \rightarrow n}\right) \\
\vdots \\
f_{\sigma}^{n-1}\left(x_{n-1}, x_{n}\right) \\
f_{\sigma}^{n}\left(x_{n}\right)
\end{array}\right)
$$

where $\sigma \in \mathcal{P}, x_{i} \in \mathbb{R}$, and $x_{i \rightarrow j}:=\left(x_{i}, x_{i+1}, \ldots, x_{j}\right)^{T} \in \mathbb{R}^{j-i+1}$ for all $1 \leq i \leq$

$j \leq n$. We stress that the dynamics of each state variable $x_{j}$ is only influenced by its own value and those of the driving states $x_{j+1 \rightarrow n}$; for each given value of the signal $\sigma$, the right-hand side of (2) is thus given by a cascade of scalar subsystems: we refer to such systems as nonlinear triangular switched systems. We make the following two assumptions, on the individual subsystems. 
${ }_{85}$ Assumption 1. For each $p \in P$, the origin of the driving system $\dot{x}_{n}=f_{p}^{n}\left(x_{n}\right)$ is globally asymptotically stable (GAS).

Assumption 2. For each $i \in\{1, \ldots, n-1\}$ and each $p \in P$ the system $\dot{x}_{i}=$ $f_{p}^{i}\left(x_{i}, x_{i+1 \rightarrow n}\right)$ is Strongly iISS with respect to the input $x_{i+1 \rightarrow n}$.

Note that the above assumptions concern only non-switched dynamics.

90 2.3. Main result

We are now ready to state our main result.

Theorem 1. Under Assumptions 1 and 2, the switched system (2) is uniformly globally asymptotically stable (UGAS).

This result is similar to [9, Theorem 1] as it provides a condition under which specific switched nonlinear systems in triangular form are UGAS. The main difference stands in the main assumption made on each stage of the cascade: here, they are required to be Strongly iISS with respect to the driving state, while in [9] they are assumed ISS. This relaxation is noteworthy as Strong iISS is much less conservative than ISS, by requiring a bounded solutions only to inputs that are below a specific threshold. For this reason, it allows us to deal with systems stabilized by saturated feedback [15] or bilinear systems [14]. We illustrate the latter in Section 3

Proof of Theorem 1. The proof is decomposed into the following five lemmas. The first one establishes the UGAS of the switched driving subsystem. Its proof is provided in Section 4.1

Lemma 1. Under Assumption 1, the driving switched system $\dot{x}_{n}=f_{\sigma}^{n}\left(x_{n}\right)$, with $\sigma \in \mathcal{P}$, is $U G A S$.

The second lemma, proved in Section 4.2, ensures the existence of the solutions of the overall switched system for all forward time. 
Lemma 2. Under Assumptions 1 and 2, for all initial state $x^{0} \in \mathbb{R}^{n}$ and all switching signal $\sigma \in \mathcal{P}$, the solution $x\left(t ; x^{0}, \sigma\right)$ of (2) exists at all $t \in \mathbb{R}_{\geq 0}$.

The third lemma, proved in Section 4.3 states that the switching between scalar systems that are ISS wrt small inputs is itself ISS wrt small inputs.

Lemma 3. Assume that, for each $p \in P$, the scalar system defined by $\dot{z}=$ ${ }_{115} g_{p}(z, u), z \in \mathbb{R}, u \in \mathbb{R}^{m}$, is ISS wrt small inputs. Then, given any signal $\sigma \in \mathcal{P}$, the switched system $\dot{z}=g_{\sigma}(z, u)$ is also ISS wrt small inputs.

The fourth lemma establishes the convergence of the solutions of the switched system to the origin. Its proof is provided in Section 4.4

Lemma 4. Under Assumptions 1 and 2, given any initial state $x^{0} \in \mathbb{R}^{n}$, the solution of the switched system [2] satisfies $\lim _{t \rightarrow+\infty}\left|x\left(t ; x^{0}, \sigma\right)\right|=0$ uniformly in the switching signal $\sigma \in \mathcal{P}$.

The last lemma states that the origin of the the switched system is stable. See Section 4.5 for its proof.

Lemma 5. Under Assumptions 1 and 2, the origin of the switched system (2) 125 is uniformly stable.

The proof of Theorem 1 readily follows from Lemmas 4 and 5 by recalling that UGAS is the combination of uniform stability and convergence of all solutions to zero uniformly in the switching signal $\sigma \in \mathcal{P}$.

\section{Switched triangular bilinear systems}

We now apply Theorem 1 to a specific class of switched triangular systems. It was shown in [14] that bilinear systems are Strongly iISS if and only if their internal dynamics is Hurwitz. We exploit this property to address the stability 
of switched triangular bilinear systems of the form:

$$
\left(\begin{array}{c}
\dot{x}_{1} \\
\vdots \\
\dot{x}_{i} \\
\vdots \\
\dot{x}_{n-1} \\
\dot{x}_{n}
\end{array}\right)=\left(\begin{array}{c}
-a_{\sigma}^{1} x_{1}+\sum_{j=2}^{n} b_{\sigma}^{1, j} x_{1} x_{j} \\
\vdots \\
-a_{\sigma}^{i} x_{i}+\sum_{j=i+1}^{n} b_{\sigma}^{i, j} x_{i} x_{j} \\
\vdots \\
-a_{\sigma}^{n-1} x_{n-1}+b_{\sigma}^{n-1, n} x_{n-1} x_{n} \\
-a_{\sigma}^{n} x_{n}
\end{array}\right)
$$

130 $b_{p}^{i, j}$ denote real constants. For this particular class of switched system, we have the following result.

Proposition 1. The switched triangular system with bilinear dynamics (3) is $U G A S$ for $\sigma \in \mathcal{P}$ if and only if $a_{p}^{i}>0$ for all $i \in\{1, \ldots, n\}$ and all $p \in P$. and that the value of the parameters $b_{p}^{i, j}$ does not affect this stability property.

Note also that, despite its triangular structure, the stability of (3) cannot be addressed by [9] as each individual subsystem $\dot{x}_{i}=-a_{p}^{i} x_{i}+\sum_{j=i+1}^{n} b_{p}^{i, j} x_{i} x_{j}$, $p \in P$ is not ISS as soon as a parameter $b_{p}^{i, j}$ is non-zero. Nonetheless, the results presented in [7, that exploits class $\mathcal{K}$ dissipation rates, could also have been used for this system.

Proof of Proposition 1. The sufficiency part of the statement results from Theorem 1 by observing that (3) can be put in the form (2) by letting, for each $p \in P, f_{p}^{i}\left(x_{i}, x_{i+1 \rightarrow n}\right)=-a_{p}^{i} x_{i}+\sum_{j=i+1}^{n} b_{p}^{i, j} x_{i} x_{j}$ for all $i=\{2, \ldots, n\}$, and 145 $f_{p}^{n}\left(x_{n}\right)=-a_{p}^{n} x_{n}$. Assumption 1 is straightforwardly satisfied as $a_{p}^{n}>0$ for each $p \in P$. Moreover, Assumption 2 can easily be checked by invoking [14, Corollary 2] since $a_{p}^{i}>0$ for all $i \in\{2, \ldots, n\}$ and all $p \in P$. To establish the necessity part, assume on the contrary that $a_{p}^{i} \geq 0$ for some $i \in\{1, \ldots, n\}$ and some $p \in P$, and consider the initial state defined as $x_{j}^{0}=0$ for all $j \in\{1, \ldots, n\} \backslash\{i\}$ ${ }_{150}$ and $x_{i}^{0}=1$ together with the switching signal $\sigma \in \mathcal{P}$ defined as $\sigma(t)=p$ for all 
$t \geq 0$. Then the solution of $(3)$ satisfies $|x(t)|=\left|x_{i}(t)\right|=e^{a_{p}^{i} t}$. Since $a_{p}^{i} \geq 0$, the state does not converge to the origin, which contradicts the UGAS property.

\section{Proofs}

\subsection{Proof of Lemma 1}

For each $p \in P$, the system $\dot{x}_{n}=f_{p}^{n}\left(x_{n}\right)$ being scalar, its GAS can be established through the same Lyapunov function $W(x)=x_{n}^{2} / 2$. In other words, the switched system $\dot{x}_{n}=f_{\sigma}^{n}\left(x_{n}\right)$ is the switching among dynamics who share a common Lyapunov function. UGAS then follows from [2, Theorem 2.1].

\subsection{Proof of Lemma 2}

160

Given any $p \in P$, the solution of the driving subsystem $\dot{x}_{n}=f_{p}^{n}\left(x_{n}\right)$ exists at all positive time by Assumption 1 In view of the regularity assumptions on $f_{p}^{n}$, this solution is also continuous over time. It follows that the system $\dot{x}_{n-1}=f_{p}^{n-1}\left(x_{n-1}, x_{n}\right)$ is a Strong iISS system with a measurable and locally bounded input. Since Strong iISS implies iISS, it follows that the solution $x_{n-1}$ exists at all positive time too. Repeating this reasoning at each stage we conclude that, for each fixed $p \in P$, the solution of the overall cascade exists at all positive time, regardless of the initial state. Consequently, the system (2) results of a switching between forward complete dynamics. Since, by assumption, only a finite number of switchings may occur over any compact time interval, we conclude that the solution of (2) exists at all positive time.

\subsection{Proof of Lemma 3}

The proof of Lemma 3 relies on the following two steps.

Claim 1: There exists $\epsilon>0$ and $\theta \in \mathcal{P} \mathcal{D}$ such that the function defined by $W(z)=z^{2} / 2$ satisfies, for all $p \in P$, all $z \in \mathbb{R}$ and all $\|d\| \leq 1$,

$$
\frac{\partial W}{\partial z}(z) g_{p}\left(z, \operatorname{sat}_{\epsilon}^{m}\left(\psi_{p}(z) d\right)\right) \leq-\theta(|z|) .
$$

where $\psi_{p}: \mathbb{R} \rightarrow \mathbb{R}_{\geq 0}$ is a continuous function satisfying $\psi_{p}(z) \geq \varphi_{p}(|z|)$ for some function $\varphi_{p} \in \mathcal{K}$. 
Claim 2: There exist $\delta \in \mathcal{K}$ and $\theta \in \mathcal{P D}$ such that, for each $p \in P$ and each $z \in \mathbb{R}$

$$
|u| \leq \delta(|z|) \quad \Rightarrow \quad \frac{\partial W}{\partial z}(z) g_{p}(z, u) \leq-\theta(|z|) .
$$

Claim 2 shows that the ISS wrt small inputs of each individual system $\dot{z}=$ $g_{p}(z, u), p \in P$, can be established through the same Lyapunov function $W(z)=$ $z^{2} / 2$. In other words, as long as the input amplitude $\|u\|$ is below an input threshold $R$, all individual systems are ISS with a common Lyapunov function. When the input amplitude is not constrained, this fact is known to ensure ISS for arbitrary switching [20, 21]. Similarly, we conclude that the switched system $\dot{z}=g_{\sigma}(z, u)$ is ISS wrt small inputs, which concludes the proof of Lemma 3 So we now proceed to proving Claims 1 and 2.

Proof of Claim 1: Consider any $p \in P$. Since $\dot{z}=g_{p}(z, u)$ is ISS wrt small inputs, there exist $\epsilon_{p}>0$ such that the system $\dot{z}=g_{p}\left(z, \operatorname{sat}_{\epsilon_{p}}^{m}(u)\right)$ is ISS. Letting $\epsilon:=\min _{p \in P} \epsilon_{p}$, it follows that $\dot{z}=g_{p}\left(z\right.$, $\left.\operatorname{sat}_{\epsilon}^{m}(u)\right)$ is ISS for each $p \epsilon$ $P$. In view of [16, Lemma 2.12], it follows that $\dot{z}=g_{p}\left(z, \operatorname{sat}_{\epsilon}^{m}(u)\right)$ is weakly robustly stable, meaning that there exists a smooth function $\psi_{p}: \mathbb{R} \rightarrow \mathbb{R}_{\geq 0}$, satisfying $\psi_{p}(z) \geq \varphi_{p}(|z|)$ for all $z \in \mathbb{R}$ with $\varphi_{p} \in \mathcal{K}_{\infty}$, such that the system $\dot{z}=g_{p}\left(z, \operatorname{sat}_{\epsilon}^{m}\left(\psi_{p}(x) d\right)\right)$ is UGAS $\mathrm{S}^{1}$ for all measurable function $d \in \mathcal{U}^{m}$ satisfying $\|d\| \leq 1$. This UGAS can be proven using the particular Lyapunov function $W(z)=z^{2} / 2$. To see this, consider the function $\tilde{\theta}_{p}: \mathbb{R}_{\geq 0} \rightarrow \mathbb{R}$ defined as

$$
\tilde{\theta}_{p}(s):=-\min \left\{z g_{p}\left(z, \operatorname{sat}_{\epsilon}^{m}\left(\psi_{p}(z) d\right)\right):|z|=s,|d| \leq 1\right\} .
$$

We claim that this function $\tilde{\theta}_{p}$ is positive definite and lower bounded by a continuous positive definite function. First, notice that $\tilde{\theta}_{p}(0)=0$ as the origin is an equilibrium point of the system $\dot{z}=g_{p}(z, 0)$. Moreover, assume that $\tilde{\theta}_{p}\left(s^{*}\right)<0$ for some $s^{*}>0$. Then, by continuity of $g_{p}$, there exists $\varepsilon>0$ such that $z g_{p}\left(z, \operatorname{sat}_{\epsilon}^{m}\left(\psi_{p}(z) d\right)\right)>0$ for all $z \in\left[(1-\varepsilon) s^{*} ;(1+\varepsilon) s^{*}\right]$ and all $|d| \leq 1$, meaning in particular that $z g_{p}(z, 0)>0$, which contradicts the fact

\footnotetext{
${ }^{1}$ Here, no switching is considered and UGAS is to be understood in the sense of [16], meaning uniformly with respect to the input $d$.
} 
that $\dot{z}=g_{p}(z, u)$ is 0-GAS (as ensured by the assumption of ISS wrt small inputs). Hence, $\tilde{\theta}_{p}(z) \geq 0$ for all $z \in \mathbb{R}$. Furthermore, assume that $\tilde{\theta}_{p}\left(s^{*}\right)=0$ for some $s^{*}>0$. Then, there exists $z^{*} \in \mathbb{R}$ and some $\left|d^{*}\right| \leq 1$ such that $g\left(z^{*}, \operatorname{sat}_{\epsilon}^{m}\left(\psi_{p}\left(z^{*}\right) d^{*}\right)\right)=0$ thus implying the existence of an equilibrium outside the origin, which in turns contradicts the UGAS of this system. In a nutshell, $\tilde{\theta}_{p}$ is a positive definite function. Now, let $\theta_{p}$ be any $\mathcal{P D}$ function satisfying $\theta_{p}(s) \leq \tilde{\theta}_{p}(s)$ and let $\theta$ be the function defined as $\theta(s):=\min _{p \in P} \theta_{p}(s)$ for all $s \in \mathbb{R}_{\geq 0}$. Then $\theta \in \mathcal{P D}$ and satisfies the statement of Claim 1.

Proof of Claim 2: Given $p \in P$ and any $u \in \mathbb{R}^{m}$, consider the perturbation defined as $d=\left(d_{1}, \ldots, d_{m}\right)^{T}$ with

$$
d_{i}=\left\{\begin{array}{cc}
0 & \text { if } z=0 \\
\frac{u_{i}}{\psi_{p}(z)} & \text { if } z \neq 0 .
\end{array}\right.
$$

Recall that $\max _{i \in\{1, \ldots, m\}}\left|u_{i}\right| \leq|u|$. Hence, under the assumption that $|u| \leq$ $\min \left\{\varphi_{p}(z) / \sqrt{m} ; \epsilon / 2\right\}$, it holds that $\left|u_{i}\right| \leq \min \left\{\psi_{p}(z) / \sqrt{m} ; \epsilon / 2\right\}$ for each $i \epsilon$ $\{1, \ldots, m\}$. It follows that $\left|d_{i}\right| \leq 1 / \sqrt{m}$, which ensures that $|d| \leq 1$. Moreover, since $\left|\psi_{p}(z) d_{i}\right| \leq\left|u_{i}\right| \leq \epsilon / 2$, it holds that $\operatorname{sat}_{\epsilon}\left(\psi_{p}(x) d_{i}\right)=\psi_{p}(x) d_{i}=u_{i}$ for each $i \in\{1, \ldots, m\}$. In other worlds, it holds that $u=\operatorname{sat}_{\epsilon}^{m}\left(\psi_{p}(x) d\right)$. To sum up we have shown that, if $|u| \leq \min \left\{\varphi_{p}(|z|) / \sqrt{m} ; \epsilon / 2\right\}$, then there exists $|d| \leq 1$ such that $u=\operatorname{sat}_{\epsilon}^{m}\left(\psi_{p}(z) d\right)$. Consequently, defining $\delta(s):=$ $\min _{p \in P}\left\{\min \left\{\varphi_{p}(s) / \sqrt{m} ; \epsilon / 2\right\}\right\}$, it is clear that $\delta \in \mathcal{K}$ and it holds that :

$$
|u| \leq \delta(|z|) \quad \Rightarrow \quad \frac{\partial W}{\partial z}(z) g_{p}(z, u) \leq-\theta(|z|)
$$

which ends the proof of Claim 2.

\subsection{Proof of Lemma 4}

Consider any $\sigma \in \mathcal{P}$ and any $x^{0} \in \mathbb{R}^{n}$ and let $x(\cdot):=x\left(\cdot ; x^{0}, \sigma\right)$ denote the solution of the switched system (2). First notice that, in view of Lemma 1 it holds that $\lim _{t \rightarrow+\infty}\left|x_{n}(t)\right|=0$ uniformly in the switching signal $\sigma \in \mathcal{P}$. Moreover, by Lemma $2 x_{n-1}(t)$ exists at all $t \in \mathbb{R}_{\geq 0}$, and Lemma 3 ensures the system $\dot{x}_{n-1}=f_{\sigma}^{n-1}\left(x_{n-1}, x_{n}\right)$ is ISS wrt small inputs $x_{n}$ : these combined 
properties ensure the converging-input converging-state property, uniformly in $\sigma \in \mathcal{P}$. It follows that $\lim _{t \rightarrow+\infty}\left|x_{n-1}(t)\right|=0$ uniformly in $\sigma \in \mathcal{P}$. Repeating this reasoning at each stage, we obtain that $\lim _{t \rightarrow+\infty}\left|x_{i}(t)\right|=0$, uniformly in $\sigma \in \mathcal{P}$, for all $i=\{1,2, \ldots, n\}$, which ends the proof of Lemma 4 .

\subsection{Proof of Lemma 5}

Given any $x^{0} \in \mathbb{R}^{n}$ and any $\sigma \in \mathcal{P}$, let $x(\cdot)$ denote the solution $x\left(\cdot ; x^{0}, \sigma\right)$ of the switched system (2). By Lemma 1] there exists $\beta_{n} \in \mathcal{K} \mathcal{L}$ such that, for any switching signal $\sigma \in \mathcal{P}$ and any initial state $x^{0} \in \mathbb{R}^{n}$, the last component of the solution of the switched system (2) satisfies

$$
\left|x_{n}(t)\right| \leq \beta_{n}\left(\left|x_{n}^{0}\right|, t\right) \leq \beta_{n}\left(\left|x^{0}\right|, 0\right), \quad \forall t \in \mathbb{R}_{\geq 0}
$$

Let $R_{n-1}$ denote the input threshold of the switched system $\dot{x}_{n-1}=f_{\sigma}^{n-1}\left(x_{n-1}, x_{n}\right)$, whose ISS wrt small inputs was shown by Lemma 3 and pick $\left|x^{0}\right|$ small enough that $\beta_{n}\left(\left|x^{0}\right|, 0\right)<R_{n-1}$. Then $\left|x_{n}(t)\right|<R_{n-1}$ at all times and consequently, from Lemma 3 , there exist $\beta_{n-1} \in \mathcal{K} \mathcal{L}$ and $\gamma_{n-1} \in \mathcal{K}_{\infty}$ such that

$$
\begin{aligned}
\left|x_{n-1}(t)\right| & \leq \beta_{n-1}\left(\left|x_{n-1}^{0}\right|, t\right)+\gamma_{n-1}\left(\left\|x_{n}\right\|\right) \\
& \leq \beta_{n-1}\left(\left|x^{0}\right|, 0\right)+\gamma_{n-1} \circ \beta_{n}\left(\left|x^{0}\right|, 0\right) .
\end{aligned}
$$

where the last inequality comes from (6). Thus, we can pick $\left|x^{0}\right|$ small enough that $\left|x_{n-1}(t)\right|<R_{n-2}$ for all $t \in \mathbb{R}_{\geq 0}$, where $R_{n-2}$ denotes the input threshold of $\dot{x}_{n-2}=f_{p}^{n-2}\left(x_{n-1}, x_{n-1 \rightarrow n}\right)$ (whose existence is ensured by Lemma 3). We stress that this choice can be made independently of the switching signal $\sigma \in \mathcal{P}$, as $\beta_{n-1}, \beta_{n}$ and $\gamma_{n-1}$ are independent of $\sigma$. Repeating this reasoning at each stage, we conclude that there exists $\Delta>0$ and $\kappa \in \mathcal{K}$ such that, for all $\sigma \in \mathcal{P}$, $|x(t)| \leq \kappa\left(\left|x^{0}\right|\right)$ for all $t \in \mathbb{R}_{\geq 0}$ and all $\left|x^{0}\right|<\Delta$, which is nothing else than the 220 uniform stability of the origin of $(2)$.

\section{References}

[1] H. Lin, P. Antsaklis, Stability and Stabilizability of Switched Linear Systems: A Survey of Recent Results, IEEE Trans. on Automatic Control 54 (2) (2009) 308-322. 
[2] D. Liberzon, Switching in Systems and Control, Systems and Control: Foundations and Applications, Birkhäuser, Boston, MA, 2003, iSBN 08176-4297-8.

[3] M. Balde, U. Boscain, P. Mason, A note on stability conditions for planar switched systems, Int. J. of Contr. 82 (10) (2009) 1882-1888.

[4] S. Dashkovskiy, B. Rueffer, F. Wirth, Small gain theorems for large scale systems and construction of ISS Lyapunov functions, SIAM Journal on Control and Optimization 48 (6) (2010) 4089-4118.

[5] E. Panteley, A. Loría, Growth rate conditions for stability of cascaded time-varying systems, Automatica 37 (3) (2001) 453-460.

[6] M. Arcak, D. Angeli, E. Sontag, A unifying integral ISS framework for stability of nonlinear cascades, SIAM J. on Contr. and Opt. 40 (2002) 8881904.

[7] H. Ito, A Lyapunov approach to cascade interconnection of Integral Inputto-State Stable systems, IEEE Trans. Autom. Control 55 (3) (2010) 702708.

[8] A. Chaillet, D. Angeli, Integral Input-to-State Stable systems in cascade, Systems \& Control Letters 57 (7) (2008) 519-527.

[9] D. Angeli, D. Liberzon, A note on uniform global asymptotic stability of nonlinear switched systems in triangular form, in: In Proc. 14th Int. Symp. on Mathematical Theory of Networks and Systems (MTNS), 2000.

[10] E. Sontag, Input to state stability: Basic concepts and results, Lecture Notes in Mathematics, Springer-Verlag, Berlin, 2006, Ch. in Nonlinear and Optimal Control Theory, pp. 163-220, p. Nistri and G. Stefani eds.

[11] E. Sontag, A. Teel, Changing supply functions in Input-to-State Stable systems, IEEE Trans. Autom. Control 40 (8) (1995) 1476-1478. 
[12] A. Chaillet, D. Angeli, H. Ito, Strong iISS is preserved under cascade interconnection, Automatica 50 (9) (2014) 2424-2427.

[13] E. Sontag, Comments on integral variants of ISS, Systems \& Control Letters 34 (1998) 93-100.

255 [14] A. Chaillet, D. Angeli, H. Ito, Combining iISS and ISS with respect to small inputs: the Strong iISS property, IEEE Trans. on Automat. Contr. 59 (9) (2014) 2518-2524.

[15] R. Azouit, A. Chaillet, L. Greco, Robustness under saturated feedback: Strong iISS for a class of nonlinear systems, in: European Control Conference, Strasbourg, France, 2014.

[16] E. Sontag, Y. Wang, On characterizations of the Input-to-State Stability property, Systems \& Control Letters 24 (1995) 351-359.

[17] D. Angeli, E. Sontag, Y. Wang, A characterization of integral input to state stability, IEEE Trans. Autom. Control 45 (2000) 1082-1097.

[18] E. Sontag, Smooth stabilization implies coprime factorization, IEEE Trans. Autom. Control 34 (4) (1989) 435-443.

[19] E. Sontag, Y. Wang, New characterizations of Input-to-State Stability, IEEE Trans. Autom. Control 41 (1996) 1283-1294.

[20] L. Vu, D. Chatterjee, D. Liberzon, Input-to-State Stability of switched systems and switching adaptive control, Automatica 43 (4) (2007) 639646.

[21] J. Mancilla-Aguilar, R. Garcia, On converse Lyapunov theorems for ISS and iISS switched nonlinear systems, Systems \& Control Letters 42 (1) (2001) $47-53$. 